COMMUNICATIONS IN

ANALYSIS AND GEOMETRY

Volume 14, Number 3, 475-496, 2006

\title{
Tableaux over Lie Algebras, Integrable Systems, and Classical Surface Theory
}

\author{
Emilio Musso And LoRenzo Nicolodi ${ }^{1}$
}

\begin{abstract}
Starting from suitable tableaux over finite dimensional Lie algebras, we provide a scheme for producing involutive linear Pfaffian systems related to various classes of submanifolds in homogeneous spaces which constitute integrable systems. These include isothermic surfaces, Willmore surfaces, and other classical soliton surfaces. Completely integrable equations such as the $G / G_{0}$-system of Terng and the curved flat system of Ferus-Pedit may be obtained as special cases of this construction. Some classes of surfaces in projective differential geometry whose Gauss-Codazzi equations are associated with tableaux over $\mathbf{s l}(4, \mathrm{R})$ are discussed.
\end{abstract}

\section{Introduction.}

It is well known that various completely integrable nonlinear PDEs (soliton equations) arise in differential geometry as compatibility conditions for the linear equations satisfied by frames adapted to submanifolds in higher dimensional homogeneous spaces. In this geometric setting, the adapted frames and the local differential invariants of a submanifold may be described as integral manifolds of an appropriate exterior differential system (EDS). The theory of EDSs has been proven to be fruitful in the study of certain soliton equations, especially as concerns their Bäcklund transformations and conservation laws. In this regard, we only mention the early work of Estabrook and Wahlquist on the Korteweg-de Vries equation and the Schrödinger equation with a cubic nonlinearity [13, 14], the work of Sasaki, Chern and his collaborators on special classes of nonlinear evolution equations $[29,11,12]$, and more recently that of Bryant, Terng and Wang on the curved flat equations [5, 33].

\footnotetext{
${ }^{1}$ Authors partially supported by the MIUR project Proprietà Geometriche delle Varietà Reali e Complesse, and by The European Contract Human Potential Programme, Research Training Network HPRN-CT-2000-00101 (EDGE)
} 
The purpose of this paper is to present a unifying approach to a number of different classes of known integrable surfaces and submanifolds in homogeneous spaces. These all ultimately derive from a special class of involutive Pfaffian differential systems. As such, the EDS point of view offers a way of better understanding the geometry of these submanifolds and yet another perspective in the search for new classes of integrable geometries.

We provide a scheme for producing linear Pfaffian systems in involution ${ }^{2}$ starting from suitable algebraic tableaux over finite dimensional Lie algebras. The interest in this construction is that several classes of submanifolds in homogeneous spaces which constitute integrable systems are related to Pfaffian systems which fit into this scheme. The solutions of these systems contain, as special cases, the solutions to well-known completely integrable equations such as the $n$-dimensional system associated with a symmetric space $G / G_{0}$ of rank $n$, the so-called $G / G_{0}$-system defined by Terng [31], and the (gauge equivalent) system for curved flats in symmetric spaces introduced by Ferus and Pedit [19]. Geometrically, besides the examples of isometric immersions of space forms into space forms, of isothermic surfaces and of $L$-isothermic surfaces, all related to curved flat systems [20, 8, 4, 27, 28], other important examples include Willmore surfaces and their counterparts in Laguerre geometry [25, 26], Godeaux-Rozet, Demoulin and asymptotically-isothermic surfaces in projective differential geometry, as well as their analogues in Lie sphere geometry $[9,15,17,18]$.

The common structure of the above examples is the concept of tableau over a Lie algebra. It takes up the familiar notion of an involutive tableau in the theory of EDSs (cf. $[23,30,6]$ ) and adapts it to the purpose. Although its simple formulation, we are not aware of it having appeared earlier in the literature. We illustrate the general procedure to associate with each tableau over a Lie algebra a Pfaffian differential system, and show that the property of being involutive for such systems can be directly detected from the properties of the corresponding tableaux. In the semisimple case, this construction applies to the $G / G_{0}$-system and the curved flat system. At the same time, we start the program of identifying the submanifold geometry associated with these systems/tableaux. This amounts to finding submanifolds in some homogeneous space whose integrability conditions are given by the Pfaffian differential system associated with a given tableau. We carry out this program for surfaces in projective differential geometry whose Gauss-Codazzi equations correspond to various tableaux over $\mathfrak{s l}(4, \mathbb{R})$.

\footnotetext{
${ }^{2}$ In the sense of Cartan-Kähler theory [6].
} 
The paper is organized as follows. In Section 2, we introduce the notion of a linear tableau over a finite dimensional Lie algebra and examine its basic properties, discussing the special case of semisimple Lie algebras. In Section 3, starting from a tableau $\mathcal{A}$ over a real Lie algebra $\mathfrak{g}$ with Lie group $G$, we construct a canonical Pfaffian exterior differential system with independence condition $(\mathcal{I}, \omega)$ on $Y=G \times \mathcal{A}$, and prove that this system is involutive in the sense of Cartan-Kähler theory. In particular, the Cartan characters of a regular flag are computed in terms of the characters of the corresponding tableau. Next, we illustrate the construction of this Pfaffian exterior differential system in the case of the $G / G_{0}$-system of Terng. In

Section 4 , we give a brief review of the moving frame method for surfaces in real projective space and explain the relation between the exterior differential systems defined by tableaux over $\mathfrak{s l}(4, \mathbb{R})$ and the equations for various classes of surfaces in projective differential geometry. We discuss the cases of Godeaux-Rozet, Demoulin, and asymptotically-isothermic surfaces. The corresponding tableaux are studied and their characters are computed.

The next step would be to develop a systematic study of tableaux over Lie algebras. This will help to answer a number of questions which naturally arise. For instance, one can ask which tableaux generate integrable differential systems and whether it is possible to formulate additional restrictions on the tableaux to guarantee the integrability. These and other questions will be the object of future investigation. We conclude by observing that the above setting is also the natural one for studying invariant variational problems, their Euler-Lagrange systems and conservation laws, in the spirit of Bryant, Griffiths and Grossman (cf. [7] and the notion of multi-contact manifold therein).

The basic reference on exterior differential systems has been [6] and our notation is consistent with this reference. The summation convention over all pairs of repeated indices in a product will be used throughout except when explicitly stated otherwise.

We thank the referees for useful comments and suggestions.

\section{Tableaux over Lie Algebras.}

\subsection{Basic Definitions.}

Let $(\mathfrak{g},[]$,$) be a finite dimensional Lie algebra (over any field of characteristic$ zero). If $\mathfrak{a}, \mathfrak{b}$ are vector subspaces of $\mathfrak{g}$ such that $\mathfrak{a} \cap \mathfrak{b}=\{0\}$, let $\mathcal{A}$ be a linear subspace of $\operatorname{Hom}(\mathfrak{a}, \mathfrak{b})$ and denote by 


$$
\rho: \operatorname{Hom}(\mathfrak{a}, \mathcal{A}) \rightarrow \mathfrak{b} \otimes \Lambda^{2}\left(\mathfrak{a}^{*}\right)
$$

the linear map defined by

$$
\rho(F)\left(A_{1}, A_{2}\right)=\frac{1}{2}\left(F\left(A_{1}\right)\left(A_{2}\right)-F\left(A_{2}\right)\left(A_{1}\right)\right),
$$

for all $F \in \operatorname{Hom}(\mathfrak{a}, \mathcal{A})$ and $A_{1}, A_{2} \in \mathfrak{a}$. The first prolongation $\mathcal{A}^{(1)} \subset$ $\operatorname{Hom}(\mathfrak{a}, \mathcal{A})$ of the subspace $\mathcal{A}$ is the kernel of $\rho$. If $\operatorname{Hom}(\mathfrak{a}, \mathfrak{b})$ is identified with $\mathfrak{b} \otimes \mathfrak{a}^{*}$, then $\mathcal{A}^{(1)}=\left(\mathcal{A} \otimes \mathfrak{a}^{*}\right) \cap\left(\mathfrak{b} \otimes S^{2}\left(\mathfrak{a}^{*}\right)\right)$, where $S^{2}\left(\mathfrak{a}^{*}\right)$ is the 2 nd symmetric power of $\mathfrak{a}^{*}$.

For any flag $\ell \in \mathcal{F}(\mathfrak{a})), \ell:(0) \subset \mathfrak{a}_{1} \subset \cdots \subset \mathfrak{a}_{k}=\mathfrak{a}$, we set

$$
\mathcal{A}_{j}(\ell)=\left\{Q \in \mathcal{A}: Q_{\mid \mathfrak{a}_{j}}=0\right\} \quad(j=1, \ldots, k) .
$$

The subspaces $\mathcal{A}_{j}$ give a filtration

$$
(0)=\mathcal{A}_{k}(\ell) \subseteq \mathcal{A}_{k-1}(\ell) \subseteq \cdots \subseteq \mathcal{A}_{0}(\ell)=\mathcal{A} .
$$

A flag $\ell$ is generic with respect to $\mathcal{A}$ if the $\operatorname{dim} \mathcal{A}_{j}(\ell)$ are a minimum, i.e.,

$$
\operatorname{dim} \mathcal{A}_{j}(\ell)=\min \left\{\operatorname{dim} \mathcal{A}_{j}(\tilde{\ell}): \tilde{\ell} \in \mathcal{F}\right\} .
$$

The set of generic flags is an open and dense subset of the flag manifold $\mathcal{F}(\mathfrak{a})$. by

The characters of $\mathcal{A}$ are the non-negative integers $s_{j}^{\prime}$ defined inductively

$$
s_{1}^{\prime}+\cdots+s_{j}^{\prime}=\operatorname{dim} \mathcal{A}-\operatorname{dim} \mathcal{A}_{j}(\ell) \quad(j=1, \ldots, k),
$$

where $\ell$ is a generic flag with respect to $\mathcal{A}$. With this formulation, one can establish the following inequality (cf. [23, 30,6])

$$
\operatorname{dim} \mathcal{A}^{(1)} \leq s_{1}^{\prime}+2 s_{2}^{\prime}+\cdots+k s_{k}^{\prime},
$$

where

$$
s_{1}^{\prime}+2 s_{2}^{\prime}+\cdots+k s_{k}^{\prime}=\operatorname{dim} \mathcal{A}+\operatorname{dim} \mathcal{A}_{1}+\cdots+\operatorname{dim} \mathcal{A}_{k-1},
$$

If equality holds in (2.3), then the space $\mathcal{A}$ is said involutive.

For $Q \in \mathcal{A}$ and for $A_{1}, A_{2} \in \mathfrak{a}$, let $R_{Q}\left(A_{1}, A_{2}\right)$ denote the equivalence class in $\mathfrak{g} / \mathfrak{a} \oplus \mathfrak{b}$ represented by $\left[A_{1}+Q\left(A_{1}\right), A_{2}+Q\left(A_{2}\right)\right] . \quad R_{Q}$ defines a $\mathfrak{g} / \mathfrak{a} \oplus \mathfrak{b}$-valued two-form on $\mathfrak{a}$ :

$$
R_{Q}:\left(A_{1}, A_{2}\right) \in \mathfrak{a} \times \mathfrak{a} \mapsto R_{Q}\left(A_{1}, A_{2}\right) \in \mathfrak{g} / \mathfrak{a} \oplus \mathfrak{b} .
$$


If $R_{Q}$ vanishes identically, we may consider the $\mathfrak{b}$-valued two-form $\tau_{Q} \in$ $\mathfrak{b} \otimes \Lambda^{2}\left(\mathfrak{a}^{*}\right)$ defined by

$\tau_{Q}\left(A_{1}, A_{2}\right):=\left[A_{1}+Q\left(A_{1}\right), A_{2}+Q\left(A_{2}\right)\right]_{\mathfrak{b}}-Q\left(\left[A_{1}+Q\left(A_{1}\right), A_{2}+Q\left(A_{2}\right)\right]_{\mathfrak{a}}\right)$, where, for $X \in \mathfrak{a} \oplus \mathfrak{b}, X_{\mathfrak{a}}\left(\right.$ resp. $X_{\mathfrak{b}}$ ) denotes the $\mathfrak{a}$ (resp. $\mathfrak{b}$ ) component of $X$.

Definition 2.1. Let $\mathfrak{g}$ be a Lie algebra and $\mathfrak{a}, \mathfrak{b}$ subspaces of $\mathfrak{g}$ as above. By a tableau over $\mathfrak{g}$ we mean a subspace $\mathcal{A}$ of $\operatorname{Hom}(\mathfrak{a}, \mathfrak{b})$ (i.e., a tableau in the usual sense) such that:

1. $R_{Q}=0$, for each $Q \in \mathcal{A}$;

2. $\tau_{Q} \in \operatorname{Im}(\rho)$, for each $Q \in \mathcal{A}$;

3. $\mathcal{A}$ is involutive.

Tableaux of this type arise naturally in the case of semisimple Lie algebras.

\subsection{Tableaux over Semisimple Lie Algebras.}

Let $\mathfrak{g}$ be a semisimple Lie algebra endowed with its Killing form $\langle$,$\rangle . Let$ $\mathfrak{g}=\mathfrak{g}_{0} \oplus \mathfrak{g}_{1}$ be a Cartan decomposition of $\mathfrak{g}$. Then $\mathfrak{g}_{0}$ is a subalgebra of $\mathfrak{g}$ and $\mathfrak{g}_{1}$ is a vector subspace such that

$$
\left[\mathfrak{g}_{0}, \mathfrak{g}_{0}\right] \subset \mathfrak{g}_{0}, \quad\left[\mathfrak{g}_{0}, \mathfrak{g}_{1}\right] \subset \mathfrak{g}_{1}, \quad\left[\mathfrak{g}_{1}, \mathfrak{g}_{1}\right] \subset \mathfrak{g}_{0} .
$$

Assume that $\mathfrak{g} / \mathfrak{g}_{0}$ have rank $k$ and that $\mathfrak{a}$ be a maximal ( $k$-dimensional) Abelian subspace of $\mathfrak{g}_{1}$. Then the subspace $\mathfrak{g}_{1}$ decomposes as $\mathfrak{g}_{1}=\mathfrak{a} \oplus \mathfrak{m}$, where

$$
\mathfrak{m}=\mathfrak{a}^{\perp} \cap \mathfrak{g}_{1}=\left\{X \in \mathfrak{g}_{1}:\langle X, A\rangle=0, \text { for all } A \in \mathfrak{a}\right\} .
$$

Further, let

$$
\begin{gathered}
\left(\mathfrak{g}_{0}\right)_{\mathfrak{a}}=\left\{X \in \mathfrak{g}_{0}:[X, \mathfrak{a}]=0\right\} \\
\left(\mathfrak{g}_{0}\right)_{\mathfrak{a}}^{\perp}=\left\{X \in \mathfrak{g}:\langle X, Y\rangle=0, \text { for all } Y \in\left(\mathfrak{g}_{0}\right)_{\mathfrak{a}}\right\}
\end{gathered}
$$

and set $\mathfrak{b}=\mathfrak{g}_{0} \cap\left(\mathfrak{g}_{0}\right)_{\mathfrak{a}}^{\perp}$. Then, for any regular ${ }^{3}$ element $A \in \mathfrak{a}$ with respect to the $\operatorname{Ad}\left(G_{0}\right)$-action on $\mathfrak{g}_{1}$, the maps

$$
\operatorname{ad}_{A}: \mathfrak{m} \rightarrow \mathfrak{b}, \quad \operatorname{ad}_{A}: \mathfrak{b} \rightarrow \mathfrak{m}
$$

\footnotetext{
${ }^{3}$ We recall that $A \in \mathfrak{g}_{1}$ is regular if the orbit at $A$ for the $\operatorname{Ad}\left(G_{0}\right)$-action is a principal orbit. If $A$ is regular, it is contained in a maximal abelian subspace $\mathfrak{a}$ in $\mathfrak{g}_{1}$ and $\operatorname{Ad}\left(G_{0}\right)(\mathfrak{a})$ is open in $\mathfrak{g}_{1}$ (cf. $\left.[4,31,33]\right)$.
} 
are vector space isomorphisms. Moreover, the mapping

$$
X \in \mathfrak{m} \mapsto-\operatorname{ad}_{X} \in \operatorname{Hom}(\mathfrak{a}, \mathfrak{b})
$$

is injective, and hence $\mathfrak{m}$ can be identified with a linear subspace of $\operatorname{Hom}(\mathfrak{a}, \mathfrak{b})$.

Proposition 2.2. If $\mathfrak{g}$ is a semisimple Lie algebra and $\mathfrak{a}, \mathfrak{b}$, and $\mathfrak{m}$ are defined as above, then $\mathfrak{m}$, regarded as a subspace of $\operatorname{Hom}(\mathfrak{a}, \mathfrak{b})$, is a tableau over $\mathfrak{g}$. It is referred to as a Cartan tableau over $\mathfrak{g}$.

Proof. First, we prove that $R_{Q}=0$, for every $Q=-\operatorname{ad}_{X}, X \in \mathfrak{m}$. For $A_{1}, A_{2} \in \mathfrak{a}$, we have

$\left[A_{1}+Q\left(A_{1}\right), A_{2}+Q\left(A_{2}\right)\right]=\left[A_{1}-\left[X, A_{1}\right], A_{2}-\left[X, A_{2}\right]\right]=\left[\left[X, A_{1}\right],\left[X, A_{2}\right]\right]$.

By Jacobi's identity,

$$
2\left[\left[X, A_{1}\right],\left[X, A_{2}\right]\right]=-\left[\left[\left[X, A_{2}\right], X\right], A_{1}\right]+\left[\left[\left[X, A_{1}\right], X\right], A_{2}\right] .
$$

Since $\left[\left[X, A_{2}\right], X\right]$ and $\left[\left[X, A_{1}\right], X\right]$ belong to $\left[\left[\mathfrak{g}_{1}, \mathfrak{g}_{1}\right], \mathfrak{g}_{1}\right] \subset\left[\mathfrak{g}_{0}, \mathfrak{g}_{1}\right] \subset \mathfrak{g}_{1}$, we can write

$$
\left[\left[X, A_{2}\right], X\right]=A^{\prime}+B^{\prime}, \quad\left[\left[X, A_{1}\right], X\right]=A^{\prime \prime}+B^{\prime \prime},
$$

for some $A^{\prime}, A^{\prime \prime} \in \mathfrak{a}$ and $B^{\prime}, B^{\prime \prime} \in \mathfrak{m}$. As a consequence,

$$
\left[\left[\left[X, A_{2}\right], X\right], A_{1}\right]=\left[B^{\prime}, A_{1}\right] \in \mathfrak{b}, \quad\left[\left[\left[X, A_{1}\right], X\right], A_{2}\right]=\left[B^{\prime \prime}, A_{2}\right] \in \mathfrak{b},
$$

which implies $\left[A_{1}+Q\left(A_{1}\right), A_{2}+Q\left(A_{2}\right)\right] \in \mathfrak{b}$, for all $A_{1}, A_{2} \in \mathfrak{a}$, and hence $R_{Q}=0$.

Next, we show that $\tau_{Q} \in \operatorname{Im}(\rho)$, for every $Q=-\operatorname{ad}_{X}, X \in \mathfrak{m}$. From the first part of the proof, we know that $\left[A_{1}+Q\left(A_{1}\right), A_{2}+Q\left(A_{2}\right)\right] \in \mathfrak{b}$ and then

$$
\tau_{Q}\left(A_{1}, A_{2}\right)=\frac{1}{2}\left(\left[\left[\left[X, A_{1}\right], X\right], A_{2}\right]-\left[\left[\left[X, A_{2}\right], X\right], A_{1}\right]\right),
$$

that is,

$$
\tau_{Q}\left(A_{1}, A_{2}\right)=\frac{1}{2}\left(\operatorname{ad}_{M_{Q}\left(A_{1}\right)}\left(A_{2}\right)-\operatorname{ad}_{M_{Q}\left(A_{2}\right)}\left(A_{1}\right)\right),
$$

where $M_{Q}$ is the liner map defined by $M_{Q}: A \in \mathfrak{a} \mapsto[[X, A], X]_{\mid \mathfrak{m}}$ (here $[[X, A], X]_{\mathfrak{m}}$ denotes the $\mathfrak{m}$ component of $\left.[[X, A], X] \in \mathfrak{g}_{1}\right)$. This shows that $\tau_{Q} \in \operatorname{Im}(\rho)$. 
Finally, we prove that $\mathfrak{m}$ is involutive. Let $\left(A_{1}, \ldots, A_{k}\right)$ be a basis consisting of regular elements of $\mathfrak{a}$. Then the flag

$$
\ell:(0) \subset \operatorname{span}\left\{A_{1}\right\} \subset \operatorname{span}\left\{A_{1}, A_{2}\right\} \subset \cdots \subset \operatorname{span}\left\{A_{1}, \ldots, A_{k}\right\}=\mathfrak{a}
$$

is generic with respect to $\mathfrak{m}$ and the characters are given by

$$
s_{1}^{\prime}=\operatorname{dim} \mathfrak{m}, \quad s_{j}^{\prime}=0 \quad(j=2, \ldots, k) .
$$

For every $X \in \mathfrak{m}$, we consider the linear map $\mu_{X}: \mathfrak{a} \rightarrow \mathfrak{m}$ defined by

$$
\left[\mu_{X}\left(A_{j}\right), A_{1}\right]=\left[X, A_{j}\right], \quad \text { for all } A_{j} .
$$

Then $\mu: X \in \mathfrak{m} \mapsto \mu_{X} \in \operatorname{Hom}(\mathfrak{a}, \mathfrak{b})$ is a linear embedding such that $\left[\mu_{X}(A), B\right]=\left[\mu_{X}(B), A\right]$, for every $X \in \mathfrak{m}$ and every $A, B \in \mathfrak{b}$. This shows that $\operatorname{Im}(\mu) \subset \mathfrak{m}^{(1)}$. In particular, we get

$$
\operatorname{dim} \mathfrak{m} \leq \operatorname{dim} \mathfrak{m}^{(1)} \leq s_{1}^{\prime}+2 s_{2}^{\prime}+\cdots+k s_{k}^{\prime}=\operatorname{dim} \mathfrak{m} .
$$

\section{The Pfaffian System Associated with a Tableau.}

Let $\mathcal{A} \subset \operatorname{Hom}(\mathfrak{a}, \mathfrak{b})$ be a tableau over a finite dimensional Lie algebra $\mathfrak{g}$ and let $G$ be a connected Lie group with Lie algebra $\mathfrak{g}$. We set $Y:=G \times \mathcal{A}$ and refer to it as the configuration space.

A basis $\left(A_{1}, \ldots, A_{k}, B_{1}, \ldots, B_{h}, C_{1}, \ldots, C_{s}\right)$ of $\mathfrak{g}$ is said to be adapted to the tableau $\mathcal{A}$ if

$$
\mathfrak{a}=\operatorname{span}\left\{A_{1}, \ldots, A_{k}\right\}, \quad \mathfrak{b}=\operatorname{span}\left\{B_{1}, \ldots, B_{h}\right\} .
$$

Moreover, we say that it is a generic adapted basis if the flag

$$
\text { (0) } \subset \operatorname{span}\left\{A_{1}\right\} \subset \cdots \subset \operatorname{span}\left\{A_{1}, \ldots, A_{k}\right\}=\mathfrak{a}
$$

is generic with respect to $\mathcal{A}$. For a generic adapted basis $\left(A_{1}, \ldots, A_{k}, B_{1}\right.$, $\left.\ldots, B_{h}, C_{1}, \ldots, C_{s}\right)$, we let

$$
\left(\alpha^{1}, \ldots, \alpha^{k}, \beta^{1}, \ldots, \beta^{h}, \gamma^{1} \ldots, \gamma^{s}\right)
$$

denote its dual coframe. For a given basis

$$
Q_{\epsilon}=Q_{\epsilon i}^{j} B_{j} \otimes \alpha^{i} \quad(\epsilon=1, \ldots m)
$$

of the tableau $\mathcal{A}$, we identify the configuration space $Y$ with $G \times \mathbb{R}^{m}$ by

$$
\left(g, p^{1}, \ldots, p^{m}\right) \in G \times \mathbb{R}^{m} \mapsto\left(g, p^{\epsilon} Q_{\epsilon}\right) \in Y .
$$


Definition 3.1. The exterior differential system associated with $\mathcal{A}$ is the Pfaffian system $(\mathcal{I}, \omega)$ on $Y$ such that $\mathcal{I}$ is generated, as exterior differential ideal, by the linearly independent 1 -forms

$$
\begin{gathered}
\eta^{j}:=\beta^{j}-p^{\epsilon} Q_{\epsilon i}^{j} \alpha^{i} \quad(j=1, \ldots, h), \\
\gamma^{1}, \ldots, \gamma^{s}
\end{gathered}
$$

with the independence condition

$$
\omega=\alpha^{1} \wedge \cdots \wedge \alpha^{k} \neq 0
$$

The Pfaffian system $(\mathcal{I}, \omega)$ is given by a filtration of subbundles $I \subset$ $J \subset T^{*} Y$ such that $J / I$ has rank $k$. The 1 -forms $\eta^{j}(j=1, \ldots, h)$ and $\gamma^{a}$ $(a=1, \ldots, s)$ span the sections of $I$, while the $\alpha^{i}(i=1, \ldots, k)$ are sections of $J$ which project to a coframe of $J / I$ at each point of $Y$.

Theorem 3.2. Let $\mathcal{A}$ be a tableau over a Lie algebra $\mathfrak{g}$. Then, the exterior differential system $(\mathcal{I}, \omega)$ on $Y$ associated with $\mathcal{A}$ is a linear Pfaffian system in involution. In particular, the characters $s_{j}^{\prime}$ of $\mathcal{A}$ coincide with the Cartan characters $s_{j}$ of the system.

Proof.

Let $V_{k}(\mathcal{I}, \omega)$ denote the set of all integral elements of dimension $k$. We remind that $(\mathcal{I}, \omega)$ is in involution at $y \in Y$ if there exists an ordinary integral element $(y, E) \in V_{k}(\mathcal{I}, \omega)(y)$ and that $(\mathcal{I}, \omega)$ is in involution if it is in involution at every point $y \in Y$. To prove the theorem, we use Cartan's test of involution for linear Pfaffian systems.

The set of 1 -forms $\left\{d p^{\epsilon}\right\}=\left\{d p^{1}, \ldots, d p^{m}\right\}$ completes $\left\{\eta^{j} ; \gamma^{a} ; \alpha^{i}\right\}$ to a local coframe of $Y$ which is adapted to $I \subset J \subset T^{*} Y$. In terms of this coframe, according to (1) of Definition 2.1, the structure equations of $(\mathcal{I}, \omega)$ become

$$
\begin{aligned}
& d \eta^{j} \equiv-Q_{\epsilon i}^{j} d p^{\epsilon} \wedge \alpha^{i}+\frac{1}{2} T_{i l}^{j} \alpha^{i} \wedge \alpha^{l} \quad \bmod \{I\} \quad(j=1, \ldots, h), \\
& d \gamma^{a} \equiv 0 \bmod \{I\} \quad(a=1, \ldots, s),
\end{aligned}
$$

where

$$
T_{i l}^{j}=-T_{l i}^{j}
$$

and $\{I\}$ denote the algebraic ideal generated by $\left\{\eta^{j} ; \gamma^{a}\right\}$. From (3.5) it follows that the Pfaffian system $(\mathcal{I}, \omega)$ is linear, i.e., $d I \equiv 0 \bmod \{J\}$. This 
is equivalent to the condition that the fibers of $V_{k}(\mathcal{I}, \omega)$ are affine linear subspaces of $G_{k}(T Y, \omega)$, with respect to the standard coordinates there. More specifically, an integral element at $y \in Y$ is defined by the equations

$$
\eta^{j}=0, \quad \gamma^{a}=0, \quad d p^{\epsilon}=p_{i}^{\epsilon} \alpha^{i},
$$

for fiber coordinates $p_{i}^{\epsilon}$ which satisfy

$$
-Q_{\epsilon i}^{j}(y) p_{i}^{\epsilon}+Q_{\epsilon i}^{j}(y) p_{j}^{\epsilon}-T_{i l}^{j}(y)=0 .
$$

Under a change of coframe

$$
\tilde{\alpha}^{i}=\alpha^{i}, \quad \tilde{\eta}^{j}=\eta^{j}, \quad \tilde{\gamma}^{a}=\gamma^{a}, \quad \tilde{\pi}^{\epsilon}=d p^{\epsilon}-x_{i}^{\epsilon} \alpha^{i},
$$

the numbers $T_{i l}^{j}(y)$ transform to

$$
\tilde{T}_{i l}^{J}(y)=-Q_{\epsilon i}^{j}(y) x_{i}^{\epsilon}+Q_{\epsilon i}^{j}(y) x_{j}^{\epsilon}-T_{i l}^{j}(y) .
$$

Then, using (2) of Definition 2.1, we may choose a coframe of the form

$$
\left\{\eta^{j} ; \gamma^{a} ; \alpha^{i} ; \pi^{\epsilon}=d p^{\epsilon}-x_{i}^{\epsilon} \alpha^{i}\right\}
$$

where $x_{i}^{\epsilon}$ are suitable smooth fuctions, so that the torsion term $T_{i l}^{j}$ in (3.5) vanishes identically and the structure equations of $(\mathcal{I}, \omega)$ take the form

$$
\begin{aligned}
d \eta^{j} & \equiv-Q_{\epsilon i}^{j} \pi^{\epsilon} \wedge \alpha^{i} \quad \bmod \{I\} & & (j=1, \ldots, h), \\
d \gamma^{a} & \equiv 0 \bmod \{I\} & & (a=1, \ldots, s) .
\end{aligned}
$$

Observe that (3.9) and (3.10) imply at once that the integral elements over $y \in Y$ form a linear space which is isomorphic to the first prolongation $\mathcal{A}^{(1)}$ of the tableaux $\mathcal{A}$.

Next, set $\pi_{i}^{j}=-Q_{\epsilon i}^{j} \pi^{\epsilon}$ and consider the tableau matrix

$$
\pi=\left(\begin{array}{c|c}
\pi_{i}^{j} & 0 \\
\hline 0 & 0
\end{array}\right)
$$

If $A_{1}, \ldots, A_{k}$ is generic with respect to $\mathcal{A}$, it is not difficult to show (cf. [6], p. 121) that

$$
s_{1}^{\prime}+\cdots+s_{j}^{\prime}=\left\{\begin{array}{l}
\text { number of independent } 1 \text {-forms } \\
\pi_{i}^{j} \text { in the first } j \text { columns of } \pi
\end{array}\right\},
$$

where $s_{1}^{\prime}, \ldots, s_{k}^{\prime}$ are the characters of the tableaux. This shows that $s_{1}^{\prime}, \ldots, s_{k}^{\prime}$ coincide with the Cartan's characters $s_{1}, \ldots, s_{k}$ of the differential 
system. Further, since $\mathcal{A}$ is involutive and since $\operatorname{dim} V_{k}(\mathcal{I}, \omega)(y)=\operatorname{dim} \mathcal{A}^{(1)}$, it follows that

$$
\operatorname{dim} V_{k}(\mathcal{I}, \omega)(y)=s_{1}+2 s_{2}+\cdots+k s_{k}, \quad \text { for each } \quad y \in Y .
$$

From Cartan's test of involution for linear Pfaffian system, we may then conclude that $(\mathcal{I}, \omega)$ is involutive, with Cartan's characters $s_{1}^{\prime}, \ldots, s_{k}^{\prime}$.

\subsection{The $G / G_{0}$-Exterior Differential System.}

Let $G / G_{0}$ be a semisimple symmetric space of rank $k$ and $\mathfrak{g}=\mathfrak{g}_{0} \oplus \mathfrak{g}_{1}$ be a Cartan decomposition of $G / G_{0}$. Let $\mathfrak{a}$ be a maximal $k$-dimensional Abelian subspace in $\mathfrak{g}_{1}$ and $\left(A_{1}, \ldots, A_{k}\right)$ a basis for $\mathfrak{a}$ consisting of regular elements with respect to the $\operatorname{Ad}\left(G_{0}\right)$-action on $\mathfrak{g}_{1}$. According to Terng [31], the $G / G_{0}$ system associated with the symmetric space $G / G_{0}$ is the following system of PDEs for maps $V: U \subset \mathfrak{a} \rightarrow \mathfrak{g}_{1} \cap \mathfrak{a}^{\perp}$ :

$$
\left[A_{i}, V_{x_{j}}\right]-\left[A_{j}, V_{x_{i}}\right]=\left[\left[A_{i}, V\right],\left[A_{j}, V\right]\right], \quad 1 \leq i \neq j \leq k,
$$

where $V_{x_{i}}=\frac{\partial V}{\partial x_{i}}$, being $x_{i}$ the coordinates with respect to $\left(A_{1}, \ldots, A_{k}\right)$.

Observe that $V: \mathfrak{a} \rightarrow \mathfrak{g}_{1} \cap \mathfrak{a}^{\perp}$ is a solution of (3.12) if and only if the $\mathfrak{g}$-valued 1-form

$$
\theta=\alpha+[\alpha, V] \in \Omega^{1}(\mathfrak{a}) \otimes \mathfrak{g},
$$

is flat, i.e., satisfies the Maurer-Cartan equation, where $\alpha=\alpha^{i} \otimes A_{i}$ denotes the tautological 1-form on $\mathfrak{a}$.

By a generalized solution of the $G / G_{0}$-system is meant a pair $(f, V)$ : $M^{k} \rightarrow \mathfrak{a} \times\left(\mathfrak{g}_{1} \cap \mathfrak{a}^{\perp}\right)$ defined on a $k$-dimensional manifold $M^{k}$ such that:

- $f: M^{k} \rightarrow \mathfrak{a}$ is a local diffeomorphism;

- the $\mathfrak{g}$-valued 1-form $d f+[d f, V] \in \Omega^{1}(\mathfrak{a}) \otimes \mathfrak{g}$ satisfies the Maurer-Cartan equation.

Remark 3.3. Note that if $(f, V)$ is a generalized solution and $U \subset M^{k}$ is an open subset such that $f_{\mid U}: U \rightarrow \mathfrak{a}$ is a diffeomorphism onto its image, then $V \circ\left(f_{\mid U}\right)^{-1}: f(U) \subset \mathfrak{a} \rightarrow \mathfrak{g}_{1} \cap \mathfrak{a}^{\perp}$ is a solution to the $G / G_{0}$-system.

Definition 3.4. Let $(f, V)$ be a generalized solution of the $G / G_{0}$-system and let $\theta$ be the corresponding 1-form. As $\theta$ satisfies the Maurer-Cartan equation, then there exists a map $g: M \rightarrow G$, uniquely defined up left multiplication by an element of $G$, such that $\theta=g^{-1} d g$ (the pull-back by $g$ 
of the Maurer-Cartan form on $G)$. $(g, V)$ is called a framed solution of the $G / G_{0}$-system.

For a map $(g, V): M^{k} \rightarrow G \times \mathfrak{g}_{1} \cap \mathfrak{a}^{\perp}$, let $\alpha$ denote the $\mathfrak{a}$ component of $g^{-1} d g$ with respect to the splitting $\mathfrak{g}=\mathfrak{g}_{0} \oplus \mathfrak{a} \oplus \mathfrak{g}_{1} \cap \mathfrak{a}^{\perp}$ of $\mathfrak{g}$. Then $(g, V)$ is a framed solution if and only if

- $g^{-1} d g=\alpha+[\alpha, V]$,

- $\alpha^{1} \wedge \cdots \wedge \alpha^{k} \neq 0$.

If $M^{k}$ is simply connected, the corresponding generalized solution is given by $(f, V)$, where $f: M^{k} \rightarrow \mathfrak{a}$ is defined by $d f=\alpha$ (note that $\alpha$ is a closed 1-form).

Definition 3.5. The $G / G_{0}$-exterior differential system is defined to be the Pfaffian system on the configuration space $Y=G \times \mathfrak{m}$ associated with the Cartan tableau $\mathfrak{m} \subset \operatorname{Hom}(\mathfrak{a}, \mathfrak{b})$.

Next, we show that the framed solutions of the $G / G_{0}$-system can be described as integral manifolds of this $G / G_{0^{-}}$exterior differential system.

Let $\mathfrak{n} \subset \mathfrak{g}_{0}$ be such that $\mathfrak{g}_{0}=\mathfrak{b} \oplus \mathfrak{n}$ and let

$$
\theta=\theta_{\mathfrak{a}}+\theta_{\mathfrak{m}}+\theta_{\mathfrak{b}}+\theta_{\mathfrak{n}}
$$

be the decomposition of the Maurer-Cartan form of $G$ with respect to the splitting

$$
\mathfrak{g}=\mathfrak{a} \oplus \mathfrak{m} \oplus \mathfrak{b} \oplus \mathfrak{n} .
$$

The Pfaffian system on $Y$ associated with the tableau $\mathfrak{m} \subset \operatorname{Hom}(\mathfrak{a}, \mathfrak{b})$ is generated by the Pfaffian equations

$$
\theta_{\mathfrak{b}}=\left[\theta_{\mathfrak{a}}, V\right], \quad \theta_{\mathfrak{m}}=\theta_{\mathfrak{n}}=0,
$$

with independence condition

$$
\theta_{\mathfrak{a}}^{1} \wedge \cdots \wedge \theta_{\mathfrak{a}}^{k} \neq 0 .
$$

The integral manifolds of this system are smooth $k$-dimensional immersed submanifolds

$$
(g, V): M^{k} \rightarrow G \times\left(\mathfrak{g}_{1} \cap \mathfrak{a}^{\perp}\right)
$$

which satisfy $g^{-1} d g=d f+[d f, V]$, where $f$ is a local diffeomorphism. This shows that the integral manifolds coincide with the framed solutions of the $G / G_{0}$-system.

Remark 3.6. According to the proof of Proposition 2.2 and Theorem 3.2, it follows that the $G / G_{0}$-system is in involution and its general solutions depend on $k$ functions in one variable (cf. [33]). 


\section{Surfaces in Projective Space and Tableaux over $\mathfrak{s l}(4, \mathbb{R})$.}

First, we briefly review some aspects of projective differential geometry of surfaces by the method of moving frames (cf. $[1,2,10,21])$.

\subsection{The Wilczynski Frame.}

Let $G=\mathrm{SL}(4, \mathbb{R}) / \mathbb{Z}_{2}$ be the full group of projective transformations of the real projective space $\mathbb{P}^{3}$. For each $g=\left(g_{0}, g_{1}, g_{2}, g_{3}\right) \in \mathrm{SL}(4, \mathbb{R})$ we shall denote by $[g]$ its equivalence class in $G$. The Lie algebra $\mathfrak{g}$ of the projective group will be identified with $\mathfrak{s l}(4, \mathbb{R})$ and the Maurer-Cartan form of $\mathfrak{g}$ will be denoted by $\theta=\left(\theta_{j}^{i}\right)=g^{-1} d g$.

Let $f: M^{2} \rightarrow \mathbb{P}^{3}$ be a smooth immersion of a connected surface. We shall still write $\theta$ instead of $f^{*}(\theta)$ to denote the pull-back of the MaurerCartan form on $M$. A projective frame field along $f$ is a smooth map $g$ : $U \rightarrow \mathrm{SL}(4, \mathbb{R})$ defined on an open set $U$ of $M$ such that $f(x)=\left[g_{0}(x)\right]$, for all $x \in U$. A projective frame along $f$ is of first order if $\theta_{0}^{3}=0$. It easily seen that first order frames exist locally near any point of $M$. Note that the 1-forms $\theta_{0}^{1}$ and $\theta_{0}^{2}$ define a coframe on $M$. Differentiating $\theta_{0}^{3}=0$, it follows, by the structure equations and Cartan's Lemma, that there exist smooth functions $h_{i j}=h_{j i}, 1 \leq i, j \leq 2$, defined on $U$ and depending on the frame field, so that

$$
\theta_{i}^{3}=h_{i j} \theta_{i}^{j}, \quad i=1,2 .
$$

The vector valued quadratic form

$$
\phi_{f}=h_{i j} \theta_{0}^{i} \theta_{0}^{j} \otimes g_{0}
$$

is independent of the choice of the first order frame and gives rise to a global cross section of the vector bundle $S^{2}(M) \otimes K_{f}$, where $S^{2}(M)$ denotes the bundle of symmetric tensor of type $(2,0)$ and $K_{f}$ is the canonical line bundle of the surface.

Definition 4.1. $\phi_{f}$ is known as the Fubini quadratic form of the surface. If $\phi_{f}$ has signature $(1,1)$, then $f: M \rightarrow \mathbb{P}^{3}$ is said to be an hyperbolic surface.

Remark 4.2. Most of the classical literature deals with surfaces of hyperbolic type. From now on we shall restrict our consideration to such surfaces.

Differentiating the equations $\theta_{i}^{3}=h_{i j} \theta_{i}^{j}(i=1,2)$ and applying the structure equations and Cartan's Lemma, we have that

$$
-d h_{i j}+h_{i j} \theta_{i}^{k}+\frac{1}{2} h_{i j}\left(\theta_{0}^{0}+\theta_{3}^{3}\right)=F_{i j k} \theta_{0}^{k},
$$


where the $F_{i j k}$ are smooth functions on $U$ symmetric in $i, j$ and $k$. The symmetric cubic form defined by

$$
F_{i j k} \theta_{0}^{i} \theta_{0}^{j} \theta_{0}^{k} \otimes g_{0}
$$

depends on the first order frame $g$ and transforms by a section $\rho \cdot \phi_{f}$ of the line subbundle $S^{1}(M) \otimes \phi_{f}$ of $S^{3}(M) \otimes K_{f}$.

Definition 4.3. The equivalence class

$$
\Psi_{f}=\left[F_{i j k} \theta_{0}^{i} \theta_{0}^{j} \theta_{0}^{k} \otimes g_{0}\right]
$$

in $S^{3}(M) \otimes K_{f} / S^{1}(M) \otimes \phi_{f}$ defines a global cross section, called the Fubini cubic form of the surface. If $\Psi_{f}$ never vanishes, then $f: M^{2} \rightarrow \mathbb{P}^{3}$ is said to be generic of hyperbolic type.

Remark 4.4. The vanishing of the Fubini cubic form characterizes hyperbolic quadrics in $\mathbb{P}^{3}$.

By successive frame reductions one can prove the classical

Theorem 4.5 (Cartan, cf. $[\mathbf{1 0}, \mathbf{2 1}, \mathbf{1}])$. If $f: M^{2} \rightarrow \mathbb{P}^{3}$ is a generic hyperbolic surface, then there exists a unique projective frame field $[\mathrm{g}]: M^{2} \rightarrow$ $G$ along $f$, referred to as the Wilczynski frame field, such that

$$
g^{-1} d g=\left(\theta_{j}^{i}\right)_{0 \leq i, j \leq 3}=\left(\begin{array}{cccc}
\theta_{0}^{0} & \theta_{1}^{0} & \theta_{2}^{0} & \theta_{3}^{0} \\
\theta_{0}^{1} & \theta_{1}^{1} & \theta_{0}^{2} & \theta_{2}^{0} \\
\theta_{0}^{2} & \theta_{0}^{1} & -\theta_{1}^{1} & \theta_{1}^{0} \\
0 & \theta_{0}^{2} & \theta_{0}^{1} & -\theta_{0}^{0}
\end{array}\right)
$$

and

$$
\theta_{0}^{1} \wedge \theta_{0}^{2} \neq 0
$$

Remark 4.6. In terms of the Wilczynski frame, the quadratic and cubic forms can be written, respectively, as:

$$
\phi_{f}=\theta_{0}^{1} \cdot \theta_{0}^{2} \otimes g_{0}, \quad \Psi_{f}=\left[\left(\theta_{0}^{1}\right)^{3}+\left(\theta_{0}^{2}\right)^{3} \otimes g_{0}\right] .
$$

The non zero components of the Maurer-Cartan form $\theta$ of the Wilczynski frame are $\theta_{0}^{1}, \theta_{0}^{2}$ (which define a coframe on $M^{2}$ ) and $\theta_{0}^{0}, \theta_{1}^{1}, \theta_{1}^{0}, \theta_{2}^{0}, \theta_{3}^{0}$. From the exterior differentiation of these forms and the structure equations, 
it follows that there exist smooth functions $q_{1}, q_{2}, p_{1}, p_{2}$, and $r_{1}, r_{2}$, the invariant functions of the surface, such that

$$
\begin{cases}\theta_{0}^{0}=-\frac{3}{2} q_{1} \theta_{0}^{1}+\frac{3}{2} q_{2} \theta_{0}^{2}, & \theta_{1}^{1}=\frac{1}{2} q_{1} \theta_{0}^{1}+\frac{1}{2} q_{2} \theta_{0}^{2}, \\ \theta_{1}^{0}=r_{1} \theta_{0}^{1}+p_{2} \theta_{0}^{2}, & \theta_{2}^{0}=p_{1} \theta_{0}^{1}+r_{2} \theta_{0}^{2}, \\ \theta_{3}^{0}=r_{2} \theta_{0}^{1}+r_{1} \theta_{0}^{2}, & \end{cases}
$$

In addition, the invariant functions must satisfy the following equations:

$$
\begin{gathered}
d \theta_{0}^{1}=-q_{2} \theta_{0}^{1} \wedge \theta_{0}^{2}, \quad d \theta_{0}^{2}=-q_{1} \theta_{0}^{1} \wedge \theta_{0}^{2}, \\
\left\{\begin{array}{l}
d q_{1} \wedge \theta_{0}^{1}-d q_{2} \wedge \theta_{0}^{2}=\frac{2}{3}\left(p_{1}-p_{2}\right) \theta_{0}^{1} \wedge \theta_{0}^{2}, \\
d q_{1} \wedge \theta_{0}^{1}+d q_{2} \wedge \theta_{0}^{2}=2\left(1+q_{1} q_{2}-p_{1}-p_{2}\right) \theta_{0}^{1} \wedge \theta_{0}^{2},
\end{array}\right. \\
\left\{\begin{array}{l}
d r_{1} \wedge \theta_{0}^{1}+d p_{2} \wedge \theta_{0}^{2}=\left(2 q_{2} r_{1}+3 q_{1} p_{2}\right) \theta_{0}^{1} \wedge \theta_{0}^{2}, \\
d p_{1} \wedge \theta_{0}^{1}+d r_{2} \wedge \theta_{0}^{2}=\left(2 q_{1} r_{2}+3 q_{2} p_{1}\right) \theta_{0}^{1} \wedge \theta_{0}^{2}, \\
d r_{2} \wedge \theta_{0}^{1}+d r_{1} \wedge \theta_{0}^{2}=4\left(q_{1} r_{1}+q_{2} r_{2}\right) \theta_{0}^{1} \wedge \theta_{0}^{2} .
\end{array}\right.
\end{gathered}
$$

Conversely, given a coframe $\left(\theta_{0}^{1}, \theta_{0}^{2}\right)$ on a simply connected surface $M$ and a set of six real-valued functions $q_{1}, q_{2}, p_{1}, p_{2}, r_{1}, r_{2}$ satisfying $(4.7),(4.8)$ and (4.9), then there exists a generic hyperbolic immersion $f: M \rightarrow \mathbb{P}^{3}$ with canonical coframe $\left(\theta_{0}^{1}, \theta_{0}^{2}\right)$ and invariant functions $q_{1}, q_{2}, p_{1}, p_{2}, r_{1}, r_{2}$.

\subsection{The Fubini-Cartan Tableau and the Corresponding Exterior Differential System.}

Let $\left(\alpha^{1}, \alpha^{2}, \beta^{1}, \ldots, \beta^{5}, \gamma^{1} \ldots, \gamma^{8}\right)$ be the basis of $\mathfrak{g}^{*}$ defined by

$$
\begin{gathered}
\alpha^{1}=\theta_{0}^{1}, \quad \alpha^{2}=\theta_{0}^{2}, \quad \beta^{1}=\theta_{0}^{0}, \quad \beta^{2}=\theta_{1}^{1}, \quad \beta^{3}=\theta_{1}^{0}, \quad \beta^{4}=\theta_{2}^{0}, \quad \beta^{5}=\theta_{4}^{0}, \\
\gamma^{1}=\theta_{3}^{0}, \quad \gamma^{2}=\theta_{0}^{2}-\theta_{1}^{3}, \quad \gamma^{3}=\theta_{0}^{1}-\theta_{2}^{3}, \quad \gamma^{4}=\theta_{1}^{1}+\theta_{2}^{2}, \\
\gamma^{5}=\theta_{0}^{1}-\theta_{1}^{2}, \quad \gamma^{6}=\theta_{0}^{2}-\theta_{2}^{1}, \quad \gamma^{7}=\theta_{1}^{0}-\theta_{3}^{2}, \quad \gamma^{8}=\theta_{2}^{0}-\theta_{3}^{1} .
\end{gathered}
$$

Let $\left(A_{1}, A_{2}, B_{1}, \ldots, B_{5}, C_{1}, \ldots, C_{8}\right)$ be its dual basis and set

$$
\mathfrak{a}=\operatorname{span}\left\{A_{1}, A_{2}\right\}, \quad \mathfrak{b}=\operatorname{span}\left\{B_{1}, \ldots, B_{5}\right\} .
$$

Definition 4.7. The Fubini-Cartan tableau is the 6-dimensional subspace $\mathcal{W} \subset \operatorname{Hom}(\mathfrak{a}, \mathfrak{b})$ consisting of all elements $Q(q, p, r)$ of the form

$$
\begin{aligned}
Q(q, p, r)=q_{1} & \left(-\frac{3}{2} B_{1}+\frac{1}{2} B_{2}\right) \otimes \alpha^{1}+q_{2}\left(\frac{3}{2} B_{1}+\frac{1}{2} B_{2}\right) \otimes \alpha^{2} \\
& +p_{1} B_{4} \otimes \alpha^{1}+p_{2} B_{3} \otimes \alpha^{2}+r_{1}\left(B_{3} \otimes \alpha^{1}+B_{5} \otimes \alpha^{2}\right) \\
& +r_{2}\left(B_{4} \otimes \alpha^{2}+B_{5} \otimes \alpha^{1}\right),
\end{aligned}
$$

where $q=\left(q_{1}, q_{2}\right), p=\left(p_{1}, p_{2}\right), r=\left(r_{1}, r_{2}\right) \in \mathbb{R}^{2}$. 
Lemma 4.8. $\mathcal{W}$ is a tableau over $\mathfrak{s l}(4, \mathbb{R})$.

Proof. For any $Q \in \mathcal{W}$, we compute

$$
\begin{gathered}
{\left[A_{1}+Q\left(A_{1}\right), A_{2}+Q\left(A_{2}\right)\right]=q_{2} A_{1}+q_{1} A_{2}+\left(p_{1}-p_{2}\right) B_{1}+\left(p_{2}+p_{1}-1\right) B_{2}} \\
-\left(q_{2} r_{1}+2 q_{1} p_{2}\right) B_{3}-\left(q_{1} r_{2}+2 q_{2} p_{1}\right) B_{4}-3\left(q_{1} r_{1}+q_{2} r_{2}\right) B_{5} .
\end{gathered}
$$

This implies that $\left[A_{1}+Q\left(A_{1}\right), A_{2}+Q\left(A_{2}\right)\right] \in \mathfrak{a} \oplus \mathfrak{b}$, for each $Q \in \mathcal{W}$, $A, B \in \mathfrak{a}$. A direct computation shows that the first prolongation $\mathcal{W}^{(1)}$ of $\mathcal{W}$ is spanned by

$$
\begin{aligned}
& Q_{1} \otimes \alpha^{1}, \quad Q_{2} \otimes \alpha^{2}, \quad Q_{3} \otimes \alpha^{1}, \quad Q_{4} \otimes \alpha^{2}, \\
& Q_{4} \otimes \alpha^{1}+Q_{5} \otimes \alpha^{2}, \quad Q_{5} \otimes \alpha^{1}+Q_{6} \otimes \alpha^{2}, \quad Q_{6} \otimes \alpha^{1}+Q_{3} \otimes \alpha^{2} .
\end{aligned}
$$

Thus $\operatorname{dim} \mathcal{W}^{(1)}=7$ and the map $\rho: \operatorname{Hom}(\mathfrak{a}, \mathcal{W}) \rightarrow \mathfrak{b} \otimes \Lambda^{2}\left(\mathfrak{a}^{*}\right)$ is surjective ${ }^{4}$

Next, consider a generic flag $\ell \in \mathcal{F}(\mathfrak{a})$ of the form

$$
\ell:(0) \subset \operatorname{span}\left\{\lambda A_{1}+\mu A_{2}\right\} \subset \mathfrak{a},
$$

for $\lambda, \mu \in \mathbb{R}, \lambda \mu \neq 0$. The linear space consisting of all $Q \in \mathcal{W}$ such that $Q_{\mid \operatorname{span}\left\{\lambda A_{1}+\mu A_{2}\right\}}=0$ is characterized by the equations

$$
q_{1}=q_{2}=0, \quad \lambda p_{1}+\mu r_{2}=\mu p_{2}+\lambda r_{1}=\mu r_{1}+\lambda r_{2}=0 .
$$

This implies that $\operatorname{dim} \mathcal{W}_{1}=1$ and therefore

$$
s_{1}^{\prime}=\operatorname{dim} \mathcal{W}-1=5, \quad s_{2}^{\prime}=6-s_{1}^{\prime}=1 .
$$

In conclusion, $\operatorname{dim} \mathcal{W}^{(1)}=7=s_{1}^{\prime}+2 s_{2}^{\prime}$, that is, $\mathcal{W}$ is involutive.

Remark 4.9. The exterior differential system defined by the Fubini-Cartan tableau is the Pfaffian differential ideal generated by the 1-forms

$$
\gamma^{1} \ldots, \gamma^{8}, \eta^{1}, \cdots, \eta^{5}
$$

where $\eta^{1}, \cdots, \eta^{5}$ are given by

$$
\begin{cases}\eta^{1}=\beta^{1}+\frac{3}{2} q_{1} \theta_{0}^{1}-\frac{3}{2} q_{2} \theta_{0}^{2}, & \eta^{2}=\beta^{2}-\frac{1}{2} q_{1} \theta_{0}^{1}-\frac{1}{2} q_{2} \theta_{0}^{2} \\ \eta^{3}=\beta^{3}-r_{1} \theta_{0}^{1}-p_{2} \theta_{0}^{2}, & \eta^{4}=\beta^{4}-p_{1} \theta_{0}^{1}-r_{2} \theta_{0}^{2} \\ \eta^{5}=\beta^{5}-r_{2} \theta_{0}^{1}-r_{1} \theta_{0}^{2} & \end{cases}
$$

\footnotetext{
${ }^{4}$ in fact, $\operatorname{dim} \operatorname{Hom}(\mathfrak{a}, \mathfrak{b})=12, \operatorname{dim}\left(\mathfrak{b} \otimes \Lambda^{2}\left(\mathfrak{a}^{*}\right)\right)=5$ and $\operatorname{dim} \operatorname{Ker} \rho=$ $\operatorname{dim} \mathcal{W}^{(1)}=7$.
} 
with independent condition $\theta_{0}^{1} \wedge \theta_{0}^{2} \neq 0$. From this we infer that the integral manifolds of the system are the 2-dimensional submanifolds

$$
(g ; q, p, r): M^{2} \rightarrow G \times \mathcal{W} \cong G \times \mathbb{R}^{6}
$$

such that

- $f=\left[g_{0}\right]: M^{2} \rightarrow \mathbb{P}^{3}$ is a generic hyperbolic surface;

- $g: M^{2} \rightarrow G$ is the Wilczynski frame along $f$;

- $q_{1}, q_{2}, p_{1}, p_{2}, r_{1}, r_{2}: M^{2} \rightarrow \mathbb{R}$ are the invariant functions of $f$.

\subsection{The Projective Gauss Map.}

We recall that the Lie quadric $Q(x)$ of the surface $f: M \rightarrow \mathbb{P}^{3}$ at $f(x)=$ $\left[y^{0}: y^{1}: y^{2}: y^{3}\right]$ is defined, with respect to the Wilczynski frame $[g(x)]$, by the equation

$$
y^{0} y^{3}-y^{1} y^{2}=0 .
$$

Thus we may represent $Q(x)$ by means of the quadratic form

$$
\chi_{f}(x)=g^{0}(x) g^{3}(x)-g^{1}(x) g^{2}(x), \quad \text { for each } \quad x \in M .
$$

Let $Q_{(2,2)}$ denote the pseudo-Riemannian symmetric space

$$
Q_{(2,2)}=\mathrm{SL}(4, \mathbb{R}) / \mathrm{SO}(2,2) .
$$

Then the 2-parameter family of Lie quadrics can be viewed as an immersion

$$
\chi_{f}: M \rightarrow Q_{(2,2)}, x \mapsto \chi_{f}(x) .
$$

This map is referred to as the projective Gauss map of the immersion $f$.

The Lie quadric $\chi_{f}(x)$ is parametrized by the map

$$
\hat{f}_{x}:(\lambda, \mu) \mapsto g_{0}(x)+\lambda g_{1}(x)+\mu g_{2}(x)+\lambda \mu g_{3}(x) .
$$

Thus the projective tangent space of $\chi_{f}(x)$ at the point $\hat{f}_{x}(\lambda, \mu)$ is the projective plane spanned by the following three linearly independent vectors

$$
\left\{\begin{array}{l}
\hat{f}_{x}(\lambda, \mu)=g_{0}(x)+\lambda g_{1}(x)+\mu g_{2}(x)+\lambda \mu g_{3}(x) \\
\partial_{\lambda} \hat{f}_{x}(\lambda, \mu)=g_{1}(x)+\mu g_{3}(x) \\
\partial_{\mu} \hat{f}_{x}(\lambda, \mu)=g_{1}(x)+\lambda g_{3}(x) .
\end{array}\right.
$$


Accordingly, the envelopes of the projective Gauss map are maps $h: M \rightarrow$ $\mathbb{R}^{4}$ of the form

$$
h=g_{0}+\lambda g_{1}+\mu g_{2}+\lambda \mu g_{3},
$$

where $\lambda, \mu$ are smooth functions, determined by the tangency condition

$$
d h \wedge \hat{f}_{x} \wedge \partial_{\lambda} \hat{f}_{x} \wedge \partial_{\mu} \hat{f}_{x}=0 .
$$

Using the Maurer-Cartan equations, we compute

$$
d h \wedge \hat{f}_{x} \wedge \partial_{\lambda} \hat{f}_{x} \wedge \partial_{\mu} \hat{f}_{x}=\left(\lambda^{2} \theta_{0}^{1}+\mu^{2} \theta_{0}^{2}-\lambda^{2} \mu^{2} \theta_{3}^{0}\right) g_{0} \wedge g_{1} \wedge \wedge g_{2} \wedge g_{3} .
$$

As $\theta_{3}^{0}=r_{2} \theta_{0}^{1}+r_{1} \theta_{0}^{2}$, then $\lambda$ and $\mu$ must be solutions of the system

$$
\lambda^{2}\left(1-\mu^{2} r_{2}\right)=0, \quad \mu^{2}\left(1-\lambda^{2} r_{1}\right)=0 .
$$

If we allow the envelopes to be imaginary, we have the classical result

Proposition 4.10 (cf. $[\mathbf{3}, \mathbf{2}, \mathbf{2 1}]$ ). The projective Gauss map of a nondegenerate hyperbolic surface $f: M \rightarrow \mathbb{P}^{3}$ has in general five envelopes; out of these, one is the surface itself, while the other four are given by

$$
h_{(\epsilon, \eta)}=g_{0}+\epsilon \sqrt{r_{2}} g_{1}+\eta \sqrt{r_{1}} g_{2}+\epsilon \eta \sqrt{r_{1} r_{2}} \mu g_{3},
$$

where $\epsilon, \eta= \pm 1$. There are two exceptional cases:

- if one of the two invariant functions $r_{1}$ or $r_{2}$ vanishes identically, say $r_{2}=0, r_{1} \neq 0$, then the projective Gauss map has three distinct envelopes: the surface itself and $h_{ \pm}=g_{0} \pm \sqrt{r_{1}} g_{2}$;

- if $r_{1}=r_{2}=0$, then there are only two envelopes: the surface itself and its dual surface $\check{f}=\left[g_{3}\right]$.

Remark 4.11. We have real envelopes if and only if $r_{1}>0$ and $r_{2}>0$.

\subsection{Godeaux-Rozet Surfaces and the Corresponding Tableau.}

Godeaux-Rozet surfaces are characterized by the fact that either $r_{1}=0$ or else $r_{2}=0$. So the projective Gauss map of a Godeaux-Rozet surface has less than five distinct envelopes. They can be viewed as integral submanifolds of the Fubini-Cartan differential system restricted to one of the two submanifolds

$$
Y_{G R}^{\prime}=\left\{(g, q, p, r) \in Y: r_{2}=0\right\}, \quad Y_{G R}^{\prime \prime}=\left\{(g, q, p, r) \in Y: r_{1}=0\right\}
$$


of $Y$. We consider $Y_{G R}^{\prime}$, the other case being analogous. It easily seen that $Y_{G R}^{\prime}$ is the configuration space of the 5 -dimensional tableau over $\mathfrak{s l}(4, \mathbb{R})$

$$
\mathcal{W}_{G R}=\left\{Q(q, p, r) \in \mathcal{W}: r_{2}=0\right\}
$$

referred to as the Godeaux-Rozet tableau. Notice that the restriction to $Y_{G R}^{\prime}$ of the Fubini-Cartan differential system is exactly the system associated with $\mathcal{W}_{G R}$. Its integral manifolds are canonical lifts of Godeaux-Rozet surfaces in 3-dimensional projective space. It is easily checked that $\mathcal{W}_{G R}$ is involutive and that its characters are

$$
s_{0}^{\prime}=13, \quad s_{1}^{\prime}=5, \quad s_{2}^{\prime}=0 .
$$

Thus a general Godeaux-Rozet surface depends on five arbitrary functions in one variable.

\subsection{Demoulin Surfaces and the Corresponding Tableau.}

Demoulin surfaces are characterized by the fact that both $r_{1}=0$ and $r_{2}=0$. So the projective Gauss map of a Demoulin surface has only two distinct envelopes. Demoulin surfaces can be viewed as integral submanifolds of the Fubini-Cartan differential system restricted to the submanifold

$$
Y_{D}=\left\{(g, q, p, r) \in Y: r_{1}=r_{2}=0\right\}
$$

$Y_{D}$ is the configuration space of the tableau over $\mathfrak{s t}(4, \mathbb{R})$

$$
\mathcal{W}_{D}=\left\{Q(q, p, r) \in \mathcal{W}: r_{1}=r_{2}=0\right\}
$$

referred to as the Demoulin tableau. The restriction to $Y_{D}$ of the FubiniCartan differential system is exactly the system associated with $\mathcal{W}_{D}$, whose integral manifolds are canonical lifts of Demoulin surfaces in 3-dimensional projective space. It is an easy exercise to check that $\mathcal{W}_{D}$ is involutive and that its characters are

$$
s_{0}^{\prime}=13, \quad s_{1}^{\prime}=4, \quad s_{2}^{\prime}=0 .
$$

Thus a general Demoulin surface depends on four arbitrary functions in one variable. 


\subsection{Asymptotically-Isothermic Surfaces.}

A surface $f: M \rightarrow \mathbb{P}^{3}$ is called asymptotically-isothermic if the 3-web defined by the families of curves satisfying the Pfaffian equations

$$
\theta_{0}^{1}=\theta_{0}^{2}=\theta_{0}^{1}+\theta_{0}^{2}=0
$$

is flat. Since the connection of this web is given by $\zeta_{w}=q_{1} \theta_{0}^{1}-q_{2} \theta_{0}^{2}$, we deduce that asymptotically-isothermic surfaces are characterized by the equation $p_{1}-p_{2}=0$. The corresponding 5 -dimensional tableau is given by

$$
\mathcal{W}_{A I}=\left\{Q\left(q, p_{1}, p_{1}, r\right) \in \mathcal{W}: t \in \mathbb{R}, q, r \in \mathbb{R}^{2}\right\} .
$$

This tableau is involutive and its characters are

$$
s_{0}^{\prime}=13, \quad s_{1}^{\prime}=5, \quad s_{2}^{\prime}=0 .
$$

Therefore, asymptotically-isothermic surfaces depend on five arbitrary functions in one variable.

Remark 4.12. More generally, one could consider the class of surfaces whose invariant functions $p_{1}$ and $p_{2}$ satisfy a linear relation, that is, are expressed by

$$
p_{1}(t)=t \cos a+b_{1}, \quad p_{2}(t)=t \sin a+b_{2},
$$

where $a, b_{1}, b_{2}$ are real constants. This class of surfaces may be viewed as an analog of linear Weingarten surfaces and include, as special examples, the surfaces of constant curvature with respect to Fubini's quadratic form $\theta_{0}^{1} \cdot \theta_{0}^{2}$. In fact, a surface $f: M \rightarrow \mathbb{P}^{3}$ has constant curvature $c$ if and only if $p_{1}+p_{2}=1+\frac{c}{2}$. Next, consider the 5 -dimensional affine tableau

$$
\mathcal{W}_{\left(a, b_{1}, b_{2}\right)}=\left\{Q\left(q, p_{1}(t), p_{2}(t), r\right): t \in \mathbb{R}, q, r \in \mathbb{R}^{2}\right\} .
$$

Notice that $\mathcal{W}_{\left(a, b_{1}, b_{2}\right)}$ is an affine subspace and as such, properly speaking, it does not fit into our scheme. Nevertheless, it is possible to develop a similar theory also for affine tableaux over Lie algebras, and then surfaces satisfying (4.14) can be viewed as integral submanifolds of the Pfaffian system on $G \times$ $\mathcal{W}_{\left(a, b_{1}, b_{2}\right)}$ associated with $\mathcal{W}_{\left(a, b_{1}, b_{2}\right)}$. Also in this case, a direct computation yields that $\mathcal{W}_{\left(a, b_{1}, b_{2}\right)}$ is involutive and that its characters are

$$
s_{0}^{\prime}=13, \quad s_{1}^{\prime}=5, \quad s_{2}^{\prime}=0 .
$$

Thus the surfaces whose invariant functions $p_{1}$ and $p_{2}$ satisfy a linear condition (including asymptotically-isothermic and constant curvature surfaces) depend on five arbitrary functions in one variable. 


\section{References.}

[1] M. A. Akivis, V. V. Goldberg, Projective differential geometry of submanifolds, North-Holland Mathematical Library, 49, North-Holland Publishing Co., Amsterdam, 1993.

[2] G. Bol, Projektive Differentialgeometrie, 2. Teil, Studia mathematica, B. IX, Vandenhoeck \& Ruprecht, Göttingen, 1954.

[3] W. Blaschke, Vorlesungen über Differentialgeometrie und geometrische Grundlagen von Einsteins Relativitätstheorie, B. 3, bearbeitet von G. Thomsen, J. Springer, Berlin, 1929.

[4] M. Brück, X. Du, J. Park, and C. L. Terng, Submanifold geometry of real Grassmannian systems, The Memoirs, vol. 155, AMS, 735 (2002), $1-95$.

[5] R. L. Bryant, Lectures given at MSRI "Integrable system seminar", 2003, unpublished notes.

[6] R. L. Bryant, S. S. Chern, R. B. Gardner, H. L. Goldschmidt, P. A. Griffiths, Exterior differential systems, Mathematical Sciences Research Institute Publications, 18, Springer-Verlag, New York, 1991.

[7] R. L. Bryant, P. A. Griffiths, D. Grossman, Exterior differential systems and Euler-Lagrange partial differential equations, Chicago Lectures in Mathematics. University of Chicago Press, Chicago, 2003.

[8] F. Burstall, U. Hertrich-Jeromin, F. Pedit, U. Pinkall, Curved flats and isothermic surfaces, Math. Z. 225 (1997), 199-209.

[9] F. Burstall, U. Hertrich-Jeromin, Harmonic maps in unfashionable geometries Manuscripta Math. 108 (2002), 171-189.

[10] É. Cartan, Sur la déformation projective des surfaces, Ann. Scient. Éc. Norm. Sup. (3) 37 (1920), 259-356; or Oeuvres Complètes, III 1, $441-538$.

[11] S. S. Chern, C. K. Peng, Lie groups and KdV equations, Manuscripta Math. 28 (1979), 207-217

[12] S. S. Chern, K. Tenenblat, Pseudospherical surfaces and evolution equations, Stud. Appl. Math. 74 (1986), 55-83. 
[13] F. B. Estabrook, H. D. Wahlquist, Prolongation structures of nonlinear evolution equations, J. Mathematical Phys. 16 (1975), 1-7.

[14] F. B. Estabrook, H. D. Wahlquist, Prolongation structures of nonlinear evolution equations. II, J. Mathematical Phys. 17 (1976), 1293-1297.

[15] E. Ferapontov, Lie sphere geometry and integrable systems, Tohoku Math. J. 52 (2000), 199-233.

[16] E. Ferapontov, Integrable systems in projective differential geometry, Kyushu J. Math. 54 (2000), 183-215.

[17] E. Ferapontov, The analogue of Wilczynski's projective frame in Lie sphere geometry: Lie-applicable surfaces and commuting Schrödinger operators with magnetic fields, Internat. J. Math. 13 (2002), 956-986.

[18] E. V. Ferapontov, W. K. Schief, Surfaces of Demoulin: differential geometry, Bäcklund transformation and integrability. J. Geom. Phys. 30 (1999), 343-363.

[19] D. Ferus, F. Pedit, Curved flats in symmetric spaces, Manuscripta Math. 91 (1996), 445-454.

[20] D. Ferus, F. Pedit, Isometric immersions of space forms and soliton theory, Math. Ann. 305 (1996), 329-342.

[21] S. P. Finikov, Projective Differential Geometry, Moscow, Leningrad, 1937.

[22] V. W. Guillemin, S. Sternberg, An algebraic model of transitive differential geometry, Bull. Amer. Math. Soc. 70 (1964), 16-47.

[23] Y. Matsushima, Sur les algèbres de Lie linéaires semi-involutives, Colloque de Topologie de Strasbourg, Décembre 1954, Université de Strasbourg.

[24] Y. Matsushima, Pseudogroupes de Lie transitifs, Séminaire Bourbaki, no. 118, Hermann, Paris, 1955.

[25] E. Musso, Deformazione di superfici nello spazio di Möbius, Rend. Istit. Mat. Univ. Trieste 27 (1995), 25-45.

[26] E. Musso, L. Nicolodi, A variational problem for surfaces in Laguerre geometry, Trans. Amer. Math. Soc. 348 (1996), 4321-4337. 
[27] E. Musso, L. Nicolodi, Isothermal surfaces in Laguerre geometry, Boll. Un. Mat. Ital. (7) II-B, Suppl. fasc. 2, 11 (1997), 125-144.

[28] E. Musso, L. Nicolodi, The Bianchi-Darboux Transform of $L$-Isothermic surfaces, Internat. J. Math. 11 (2000), 911-924.

[29] R. Sasaki, Soliton equations and pseudospherical surfaces, Nuclear Phys. B 154 (1979), 343-357.

[30] I. M. Singer, S. Sternberg, The infinite groups of Lie and Cartan. I. The transitive groups, J. Analyse Math. 15 (1965), 1-114.

[31] C. L. Terng, Soliton equations and differential geometry, J. Differential Geom. 45 (1997), 407-445.

[32] C. L. Terng, Geometries and symmetries of soliton equations and integrable elliptic systems, to appear in Surveys on Geometry and Integrable Systems, Advanced Studies in Pure Mathematics, Mathematical Society of Japan.

[33] C. L. Terng, E. Wang, Curved Flats, exterior differential systems and conservation laws; to appear in Complex, Contact and Symmetric Manifolds. in honor of L. Vanhecke, Progress in Mathematics, Vol. 234, O. Kowalski; E. Musso; D. Perrone (Eds.), Birkhäuser, 2005.

Lorenzo Nicolodi

Dipartimento di Matematica

Università degli Studi di Parma

Viale G. P. Usberti 53/A

I-43100 Parma

Italy

Emilio Musso

Dipartimento di Matematica Pura ed Applicata

Università degli Studi dell'Aquila

Via Vetoio, loc. Coppito

I-67010 L'Aquila

Italy 